\title{
Mapping the Future of Particle Radiobiology in Europe: The INSPIRE Project
}

\section{OPEN ACCESS}

Edited by:

Federico Giove,

Centro Fermi - Museo storico della fisica e Centro studi e ricerche Enrico

Fermi, Italy

Reviewed by:

Michela Marafini,

Centro Fermi - Museo storico della fisica e Centro studi e ricerche Enrico

Fermi, Italy

Giuseppe A. Pablo Cirrone,

Laboratori Nazionali del Sud

(INFN), Italy

*Correspondence:

Marco Durante

m.durante@gsi.de

tThese authors have contributed equally to this work

Specialty section:

This article was submitted to Medical Physics and Imaging,

a section of the journal

Frontiers in Physics

Received: 23 May 2020

Accepted: 02 September 2020

Published: 06 October 2020

Citation:

Henthorn NT, Sokol O, Durante M, De

Marzi L, Pouzoulet F, Miszczyk J,

Olko P, Brandenburg S, van

Goethem MJ, Barazzuol L, Tambas M,

Langendijk JA, Davídková M,

Vondráĉek $V$, Bodenstein $E$

Pawelke J, Lomax AJ, Weber DC,

Dasu A, Stenerlöw B, Poulsen PR,

Sørensen BS, Grau C, Sitarz MK,

Heuskin A-C, Lucas S,

Warmenhoven JW, Merchant MJ,

Mackay RI and Kirkby KJ (2020)

Mapping the Future of Particle Radiobiology in Europe: The INSPIRE

Project. Front. Phys. 8:565055.

doi: 10.3389/fphy.2020.565055
Nicholas T. Henthorn ${ }^{1,2 \dagger}$, Olga Sokol ${ }^{3 t}$, Marco Durante ${ }^{3,4 *}$, Ludovic De Marzi ${ }^{5}$, Frederic Pouzoulet ${ }^{6}$, Justyna Miszczyk ${ }^{7}$, Pawel Olko ${ }^{7}$, Sytze Brandenburg ${ }^{8,9}$, Marc Jan van Goethem ${ }^{8,9}$, Lara Barazzuol ${ }^{9,10}$, Makbule Tambas ${ }^{9}$, Johannes A. Langendijk ${ }^{9}$, Marie Davídková ${ }^{11}$, Vladimír Vondráĉek ${ }^{12}$, Elisabeth Bodenstein ${ }^{13}$, Joerg Pawelke ${ }^{13,14}$, Antony J. Lomax ${ }^{15,16}$, Damien C. Weber ${ }^{15,17,18}$, Alexandru Dasu ${ }^{19,20}$, Bo Stenerlöw ${ }^{20}$, Per R. Poulsen ${ }^{21}$, Brita S. Sørensen ${ }^{21}$, Cai Grau ${ }^{21}$, Mateusz K. Sitarz ${ }^{21}$, Anne-Catherine Heuskin ${ }^{22}$, Stephane Lucas ${ }^{22}$, John W. Warmenhoven ${ }^{1,2}$, Michael J. Merchant ${ }^{1,2}$, Ran I. Mackay ${ }^{1,23}$ and Karen J. Kirkby ${ }^{1,2}$

${ }^{1}$ Division of Cancer Sciences, Faculty of Biology, Medicine and Health, School of Medical Sciences, The University of Manchester, Manchester, United Kingdom, ${ }^{2}$ Manchester Academic Health Science Centre, The Christie NHS Foundation Trust, Manchester, United Kingdom, ${ }^{3}$ Department of Biophysics, GSI Helmholtzzentrum für Schwerionenforschung, Darmstadt, Germany, ${ }^{4}$ Department of Condensed Matter Physics, Technische Universität Darmstadt, Darmstadt, Germany, ${ }^{5}$ Radiation Oncology Department, Institut Curie, PSL Research University, Paris, France, ${ }^{6}$ Translational Research Department, Experimental Radiotherapy Platform, Institut Curie, PSL Research University, Orsay, France, ${ }^{7}$ Department of Experimental Physics of Complex Systems, Institute of Nuclear Physics Polish Academy of Sciences, Krakow, Poland, ${ }^{8} \mathrm{KVI}$-Center for Advanced Radiation Technology, University of Groningen, Groningen, Netherlands, ${ }^{9}$ Department of Radiation Oncology, University Medical Center Groningen, University of Groningen, Groningen, Netherlands, ${ }^{10}$ Section of Molecular Cell Biology, Department of Biomedical Sciences of Cell and Systems, University Medical Center Groningen, University of Groningen, Groningen, Netherlands, ${ }^{11}$ Department of Radiation Dosimetry, Nuclear Physics Institute of the CAS, Prague, Czechia, ${ }^{12}$ Proton Therapy Center Czech, Prague, Czechia, ${ }^{13}$ OncoRay - National Center for Radiation Research in Oncology, Faculty of Medicine and University Hospital Carl Gustav Carus, Helmholtz-Zentrum Dresden - Rossendorf, Technische Universität Dresden, Dresden, Germany, ${ }^{14} \mathrm{Helmholtz-Zentrum} \mathrm{Dresden} \mathrm{-} \mathrm{Rossendorf,} \mathrm{Institute} \mathrm{of} \mathrm{Radiooncology}$ - OncoRay, Dresden, Germany, ${ }^{15}$ Centre for Proton Therapy, Paul Scherrer Institute, Villigen, Switzerland, ${ }^{16}$ Department of Physics, ETH, Zurich, Switzerland, ${ }^{17}$ Department of Radiation Oncology, University of Zurich, Zurich, Switzerland, ${ }^{18}$ Department of Radiation Oncology, University of Bern, Bern, Switzerland, ${ }^{19}$ The Skandion Clinic, Uppsala, Sweden, ${ }^{20}$ Department of Immunology, Genetics and Pathology, Uppsala University, Uppsala, Sweden, ${ }^{21}$ Danish Center for Particle Therapy, Aarhus University Hospital, Aarhus, Denmark, ${ }^{22}$ LARN-Laboratory, Namur Research Institute for Life Sciences (NARILIS), University of Namur, Namur, Belgium, ${ }^{23}$ Christie Medical Physics and Engineering, The Christie NHS Foundation Trust, Manchester, United Kingdom

Particle therapy is a growing cancer treatment modality worldwide. However, there still remains a number of unanswered questions considering differences in the biological response between particles and photons. These questions, and probing of biological mechanisms in general, necessitate experimental investigation. The "Infrastructure in Proton International Research" (INSPIRE) project was created to provide an infrastructure for European research, unify research efforts on the topic of proton and ion therapy across Europe, and to facilitate the sharing of information and resources. This work highlights the radiobiological capabilities of the INSPIRE partners, providing details of physics (available particle types and energies), biology (sample preparation and post-irradiation analysis), and researcher access (the process of applying for beam time). The collection of information reported here is designed to provide researchers both in Europe and worldwide with the tools required to select the optimal center for their research needs. We also highlight areas of redundancy in capabilities and suggest areas for future investment.

Keywords: proton therapy, radiotherapy, radiobiology, beamline, irradiation 


\section{INTRODUCTION}

There is a growing investment in proton and heavy ion therapy worldwide, with 89 proton centers and 12 carbon centers currently in clinical operation [according to the Particle Therapy Co-Operation Group (PTCOG)] [1]. Of these worldwide facilities, 31 proton centers $(\sim 35 \%)$ and four carbon centers $(\sim 33 \%)$ are located in Europe [2]. Despite the increasing adoption of particle therapy there remains a number of unanswered questions about this relatively new treatment modality [3]. These questions range widely in scope and include physical (e.g., range uncertainties or organ motion), biological (e.g., uncertainties in relative biological effectiveness and lack of clinically relevant in vivo data), and societal aspects (e.g., cost-effectiveness and radiotherapy demand) [4]. Many clinical centers offer beam time for research activities to address some of these questions [5]. However, access and utilization of this beam time can be difficult due to a lack of supply and/or funding. Rectifying this situation requires targeted efforts from both researchers and funders alike.

The European project "Infrastructure in Proton International Research" (INSPIRE) was created to allow researchers across Europe access to "state-of-the-art" research capabilities in centers for proton therapy. In addition, multi-ion research centers (research facility of UMCG, Groningen, the Netherlands; GSI, Darmstadt, Germany) augment the particle research portfolio. INSPIRE aims to integrate research activities in protons (and heavy ions) across Europe through eight objectives:

1) Developing new infrastructure by bringing together clinical, academic, and industrial research activities.
2) Enabling access to research infrastructure for researchers in both the public and private sector.

3) Providing training for the next generation of researchers in the field.

4) Facilitating knowledge exchange to promote best research practices throughout Europe.

5) Developing joint research activities (JRAs) that will improve the facilities available within the infrastructure.

6) Developing JRAs in fields where technological challenges exist to improve European competitiveness.

7) Developing an innovation pipeline to translate research into clinical practice and industrial products.

8) To conduct research within the principles of responsible research and innovation.

The project is comprised of 17 European partners, 11 of which offer beam time through transnational access (TNA) (Table 1); a complete list of the INSPIRE partners can be found at https:// protonsinspire.eu/. Further to the partners discussed in this work, the University of Namur (Belgium) is also an INSPIRE partner taking part in radiobiological research, but with their nearby partner center under development does not offer TNA through INSPIRE. However, once operational their resources will be available outside of the current INSPIRE project. Most of these partners are either clinical centers or have very close connections to clinical centers (Figure 1), for example the radiobiological capabilities of CHRISTIE and UNIMAN are shared. A close clinical link is essential to aid the design of the research at inception and to ensure its relevance and future translation to the clinic.

TABLE 1 | The INSPIRE partners offering equipment and support for radiobiological experiments through transnational access.

\begin{tabular}{|c|c|c|c|}
\hline Center & Abbreviation & Location & Website \\
\hline Aarhus University & $\mathrm{AU}$ & Aarhus, Denmark & $\begin{array}{l}\text { https://www.en.auh.dk/departments/the-danish-centre-for- } \\
\text { particle-therapy/ }\end{array}$ \\
\hline The Christie NHS foundation trust & CHRISTIE & Manchester, UK & https://www.christie.nhs.uk \\
\hline $\begin{array}{l}\text { GSI Helmholtz center for heavy lon } \\
\text { research }\end{array}$ & GSI & Darmstadt, Germany & https://www.gsi.de/work/forschung/biophysik.htm \\
\hline $\begin{array}{l}\text { The Henryk Niewodniczański institute of } \\
\text { nuclear physics polish academy of } \\
\text { sciences }\end{array}$ & IFJ PAN & Kraków, Poland & $\begin{array}{l}\text { https://inspire.ifj.edu.pl/en/index.php/dostep-do- } \\
\text { infrastruktury-badawczej/ }\end{array}$ \\
\hline Curie institute & Institut curie & Paris, France & $\begin{array}{l}\text { https://institut-curie.org/page/research-and-development- } \\
\text { proton-therapy-center }\end{array}$ \\
\hline $\begin{array}{l}\text { Nuclear physics institute of the Czech } \\
\text { academy of sciences }\end{array}$ & NPI-CAS & Prague, Czech Republic & http://www.ujf.cas.cz/en/ \\
\hline Paul Scherrer institute & PSI & Zurich, Switzerland & https://www.psi.ch/en \\
\hline Skandion clinic & Skandion & Uppsala, Sweden & https://skandionkliniken.se/ \\
\hline Technical University of Dresden & TUD & Dresden, Germany & https://www.oncoray.de/research/offer-for-users/ \\
\hline University medical center Groningen & UMCG & Groningen, Netherlands & $\begin{array}{l}\text { Clinical facility: } \\
\text { https://www.umcgradiotherapie.nl/en/umc-groningen- } \\
\text { department-of-radiation-oncology } \\
\text { Research facility: } \\
\text { https://www.rug.nl/kvi-cart/research/facilities/agor/ }\end{array}$ \\
\hline University of Manchester & UNIMAN & Manchester, UK & $\begin{array}{l}\text { https://www.bmh.manchester.ac.uk/research/domains/ } \\
\text { cancer/proton/ }\end{array}$ \\
\hline
\end{tabular}




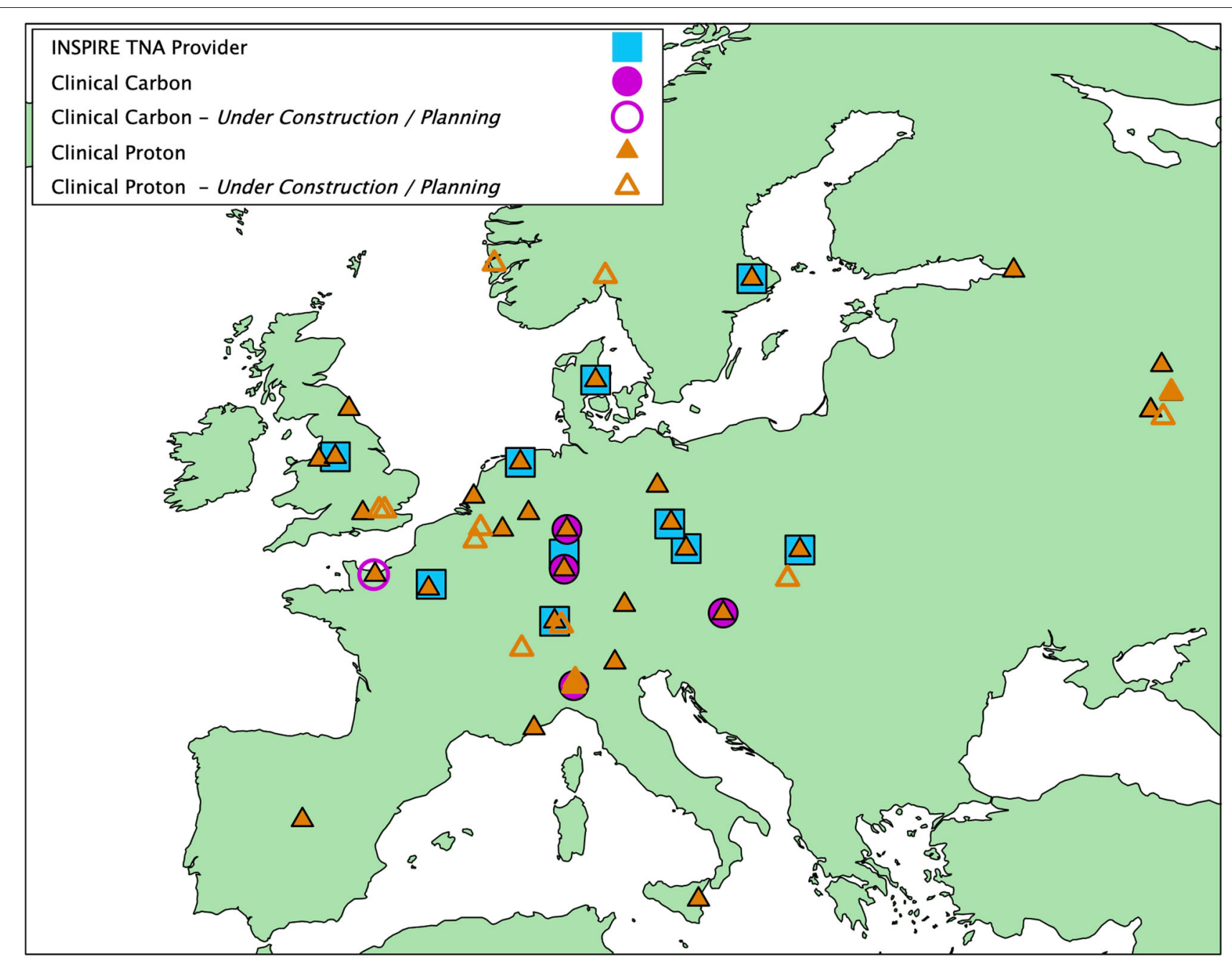

FIGURE 1 | European clinical proton therapy centers (closed triangle, 26 centers), carbon therapy centers (closed circle, 4 centers), and INSPIRE partners offering radiobiological TNA (closed squares, 11 centers - there is some overlap between centers). Open symbols show centers currently in the planning stage or under construction. Information is from the PTCOG website [1].

Further to the information hosted by each institute's website, and the information presented in this work, the following references give more information and available setups for Institut Curie [6-8], TUD [9-16], IFJ PAN [17], UMCG [18-24], and GSI [25-31].

Through INSPIRE we are able to investigate important research questions together and benefit from cross-validation. An immediate example is the variability in data for proton relative biological effectiveness (RBE) that has been seen in the literature over the years [32-35]. A coordinated effort amongst the INSPIRE partners is allowing this variability to be investigated both computationally and experimentally, and the results made available to researchers across Europe through INSPIRE's experimental and modeling JRA. This systematic and coordinated approach will highlight factors leading to variation and propose mitigation strategies for future studies. These mitigation strategies will help to develop best practices for proton radiobiology research and build upon previous work on the topic [36]. Alongside coordinated research INSPIRE also seeks to improve the infrastructure available to European researchers through its TNA. Many research centers have invested significantly to develop their research, constructing accelerators, beamlines, and purchasing experimental equipment. INSPIRE also continually upgrades its research capabilities by taking research developed through JRA and making it available to the wider research community via TNA. This means that INSPIRE is able to offer the very latest technology and capabilities.

TNA provides researchers an opportunity to access beam time and funding for experiments at INSPIRE partners. The beam time is offered to all researchers and is not limited to INSPIRE partners. Furthermore, whilst the beam time is largely accessible for European researchers, up to $30 \%$ of the hours are available to researchers outside the EU. The application process 
is managed through the INSPIRE website (https://protonsinspire. eu). Prior to submitting the application through the online form, the researchers are advised to contact the representative of the relevant partner site to discuss the technical details of their proposed experiment. Before being transferred to an independent international user selection panel (USP), the refined application, submitted via the online form, is first assessed to ensure that the requested TNA site has the capacity and infrastructure to perform the experiment. Afterwards, the application is evaluated by at least two members of the USP for its technical and scientific excellence, as well as future potential and impact. Priority is given to users who have not had access to the TNA before. The INSPIRE website contains details about each center, links to websites, and contact information for general enquiries aimed to aid the potential researcher.

The information provided in this paper acts as a corollary to the INSPIRE website, where up-to-date information is maintained. Here, we provide details of the TNA radiobiology capabilities of each INSPIRE partner. Similar information, at least in terms of the physics capabilities, has previously been presented by the European Particle Therapy Network [37] and can be used alongside this work. Planning of a radiobiological experiment requires the knowledge of not only the beamline for the sample irradiation, but also of the available equipment and capabilities of the biological laboratories on site. The latter are essential for the sample preparation and post-processing. In this work, we aim to provide comprehensive information on the facilities available across INSPIRE. We specify details of the "physics," including location, beamlines, particle types, energies, and field sizes. We specify details of the "logistics," including details of sample types, positioning, and automation. We specify details of the "biology," including the available equipment for sample preparation and post-irradiation processing. Finally, we discuss future perspectives for ongoing development and further investment. The details provided here act as a resource for the potential researcher to select the optimal center for their experimental needs. However, it should be noted that there is often flexibility in many of the aspects we report. As such the information we provide should be used as a guide and more specific details can be obtained through communication with a specific partner or through INSPIRE's help desk. It is apparent that the capabilities, at least in terms of "physics," between many partners are similar. This level of redundancy is desirable, enabling repetition to ensure scientific rigor, however, establishing these centers requires a large investment and

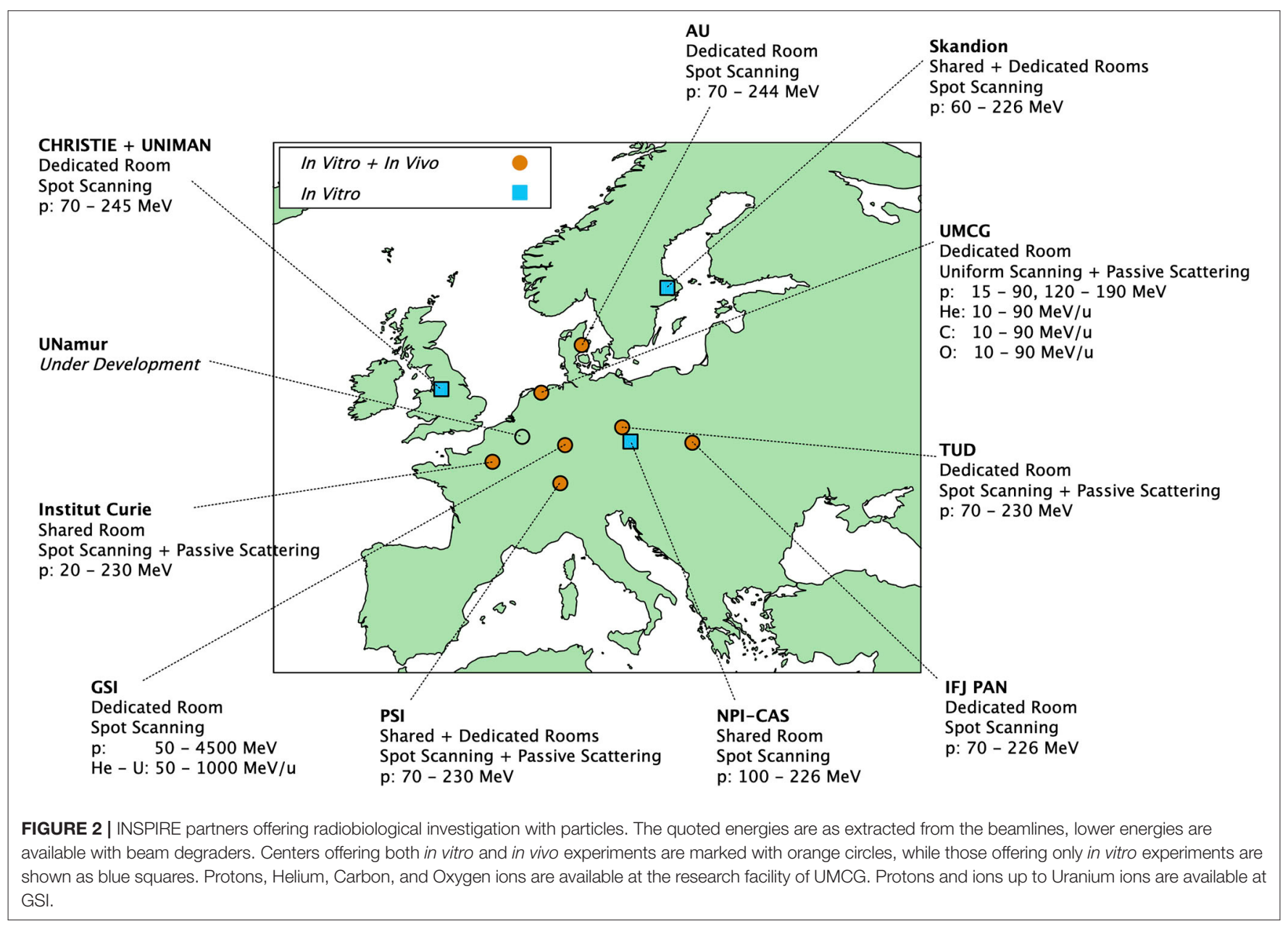




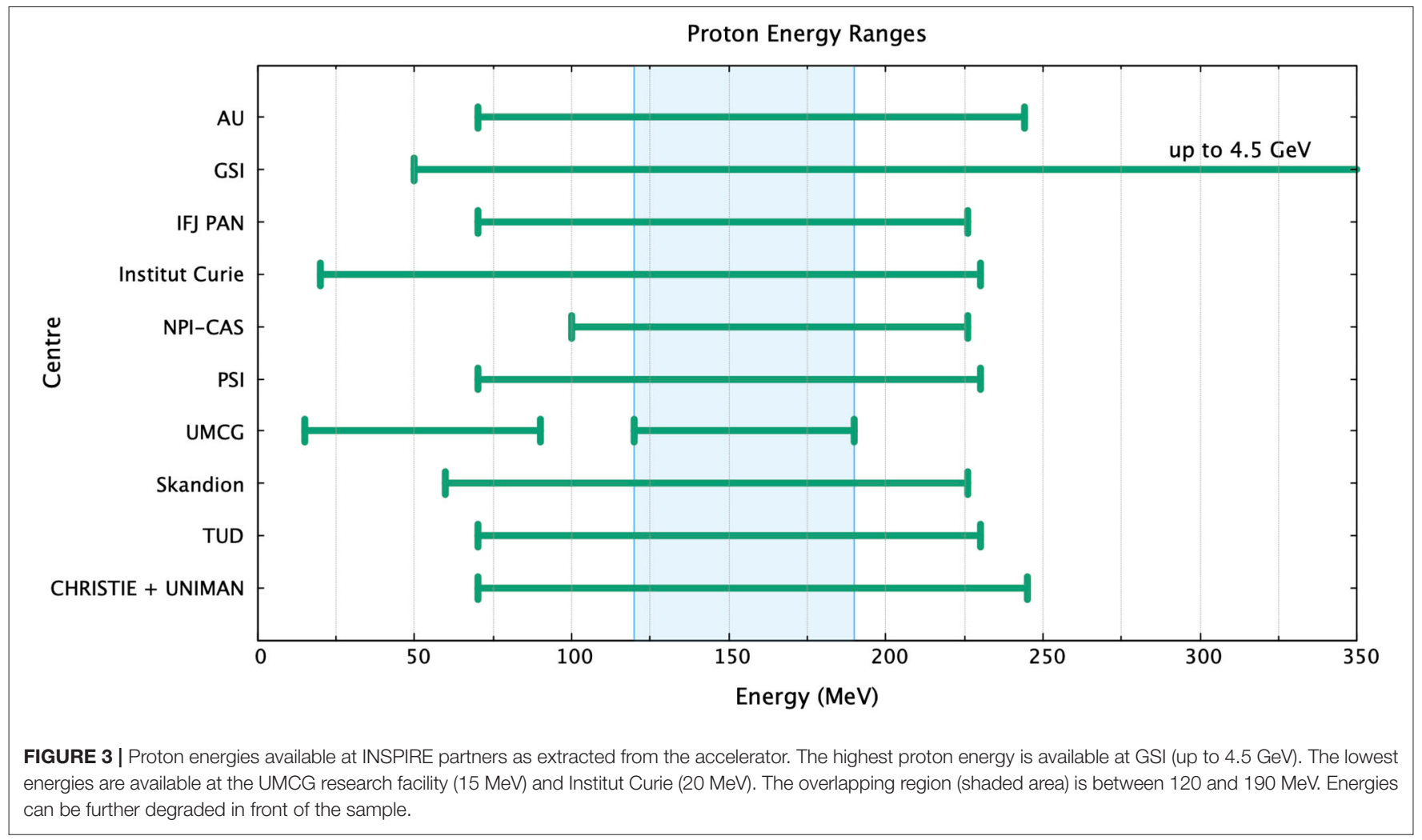

through INSPIRE they are able to work effectively together to ensure optimum utilization.

\section{PHYSICS - LOCATION, BEAMLINES, PARTICLES, ENERGIES, AND FIELDS}

A researcher often faces large heterogeneity when performing experiments between centers, with differences in protocol, setup, irradiation, and sample processing. Despite this there are a number of overlaps in beam properties and possible experiments between centers. Figure 2 shows a summary of capabilities for the INSPIRE TNA partners.

TNA providers mainly cover central and northern Europe, with a similar distribution to clinical centers (Figure 1). Geographic positioning of centers is an important factor to minimize both travel expenses and logistics. A new initiative with the South East European International Institute for Sustainable Technologies (SEEIST) [38, 39] aims to enable researchers from the south east of Europe to access INSPIRE's capabilities while they are developing their own facilities.

All of the TNA providers can supply protons, with two centers, GSI and the research facility of UMCG, additionally offering other ion types of clinical interest, such as carbon, helium, or oxygen. As can be seen from Figure 3, in general, the energies available from the accelerator are similar between providers. The most overlapping energy region is between 120 and $190 \mathrm{MeV}-$ experiments at this energy can be done at all of the partner centers. The highest possible energies can be achieved at GSI, reaching up to $1 \mathrm{GeV} / \mathrm{u}$ for heavy ions and $4.5 \mathrm{GeV} / \mathrm{u}$ for protons, with relevance to proton radiography [40] experiments, while most of the other institutes are limited to a maximum of 230-240 MeV/u. The lowest possible proton energies are offered at the research facility of UMCG (15 MeV) and Institut Curie $(20 \mathrm{MeV})$. Energies can be further degraded before the sample to investigate increased proton linear energy transfer, with a relevance for end of range effects. Access to even lower energies can be obtained through the EU project RADIATE [41].

Eight TNA providers have a dedicated research room. This can be useful for studies that require longer irradiations and/or longer follow-up, it also gives more freedom to experiments that require a complex or non-standard sample setup. However, the cost of such studies should always be considered. Whilst the sample may be able to remain in the room post-irradiation this will often inactivate the room using valuable resources. A shared room has the downside of limited usage, due to clinical commitments, although it has the added benefit of rigorous quality assurance to a clinical standard. However, it should be noted that all partners undertake measures to ensure dosimetry and quality of beam delivery in their research rooms.

Figure 4 shows examples of beamlines for the CHRISTIE + UNIMAN, Skandion, the research facility of UMCG, TUD, GSI, and Institut Curie partners.

There is a range of maximum available scanned field sizes across the INSPIRE partners, shown in Figure 5. Six partners, PSI, Skandion, NPI-CAS, IFJ PAN, AU, and Institut Curie, offer the same field size $\left(30 \times 40 \mathrm{~cm}^{2}\right)$. TUD and CHRISTIE + UNIMAN offer the same field size but in the landscape 

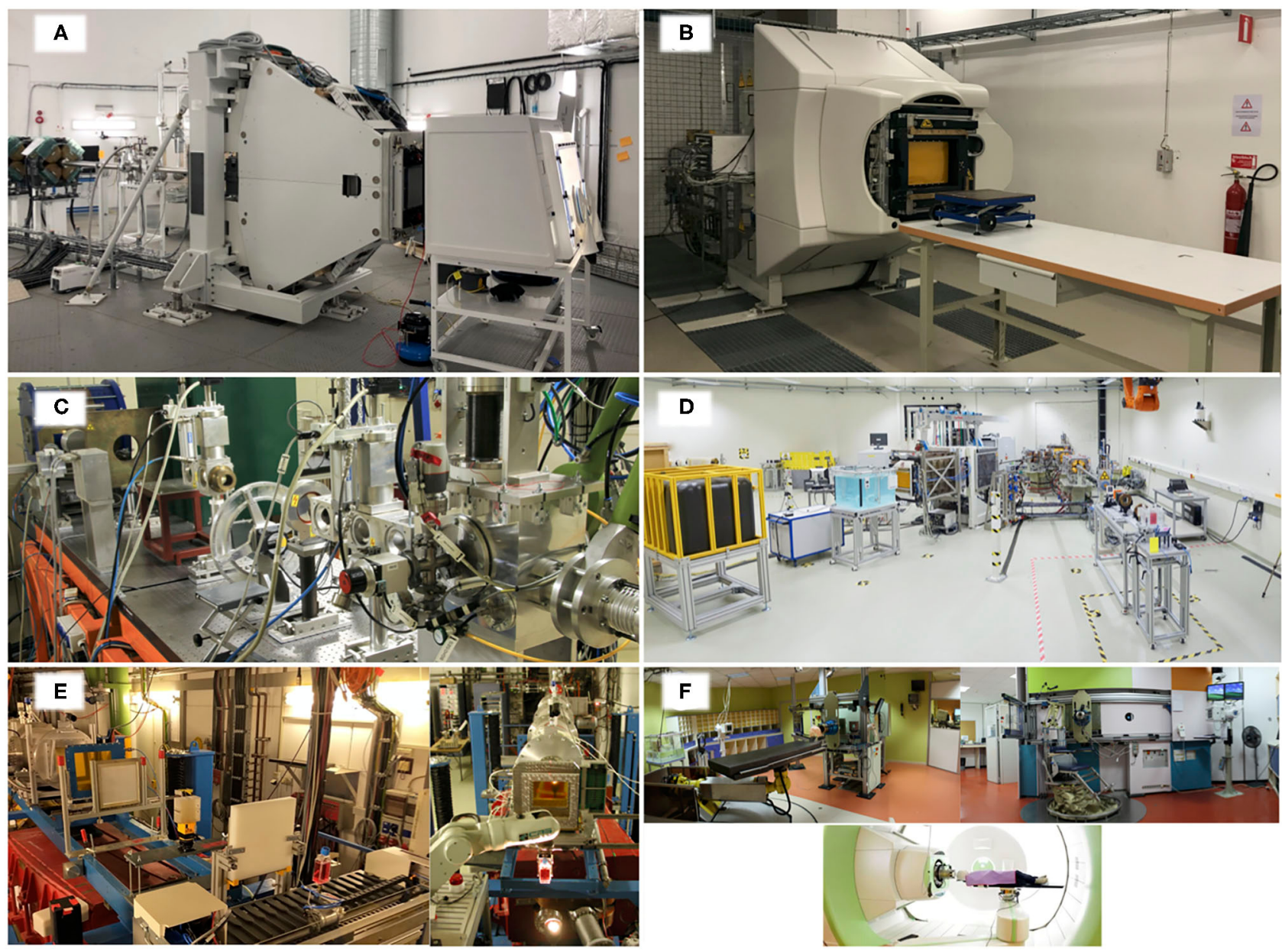

FIGURE 4 | Beamline setup for (A) UNIMAN + CHRISTIE, (B) Skandion, (C) the research facility of UMCG, (D) TUD, (E) GSI, and (F) Institut Curie. UNIMAN has a beamline leading to a Varian scanning nozzle, samples are placed in front of the nozzle (pictured is a hypoxia cabinet). Skandion has a beamline leading to an IBA scanning nozzle, samples are placed on an adjustable table in front of the nozzle. The research facility of UMCG has a flexible beamline setup using optical benches; picture shows a study on the effect of magnetic fields in combination to proton irradiation [23]. TUD has two beamlines in the dedicated experimental room, one with a pencil beam scanning nozzle (left) and one static beamline (right). In the picture, setups with water tank and beam dump at the scanning beamline and passive double scattering setup for radiobiological experiments at the static beamline are shown. GSI shows the beamline setup for "Cave A," equipped with the robotic system for sample exchange. Institut Curie shows three irradiation rooms; "Room Y1" - horizontal beam up to 201 MeV (left), "Room Y2" - horizontal beam up to 76 MeV (right), and "IBA Room" - gantry up to $230 \mathrm{MeV}$ (bottom).

orientation $\left(40 \times 30 \mathrm{~cm}^{2}\right)$. All partners offer a field size large enough to irradiate most in vitro sample types, such as tissue culture flasks or microplates. The field size may become a limitation for larger non-standard samples, or simultaneous irradiation of multiple samples. Though in some cases the field size may be increased by introducing scatterers.

Choice of reference radiation is an important aspect in general for radiobiology. The biological effects of protons are often quoted relative to the more familiar photon case, most notably the relative biological effectiveness for cell kill. A variety of reference photon qualities are used between the INSPIRE partners. Several partners have the possibility to choose between clinical LINACs and kilovoltage X-ray machines (CHRISTIE + UNIMAN, TUD, NPI-CAS, Institut Curie, UMCG), whilst the capabilities of others are more limited. The difference in reference radiation may lead to slight differences in relative effect measurements, making inter-center comparisons more complicated. However, it should be noted that this is a problem for radiobiology in general and is not limited to INSPIRE partners [42].

\section{LOGISTICS - SAMPLES, POSITIONING, AND AUTOMATION}

The mode of sample irradiation is an important consideration, including sample orientation and possibility of automated handling. Monolayers of cells, grown in a flask or microplate, should not be free from media for a long duration of time to avoid drying. As such, several centers, particularly with horizontal 


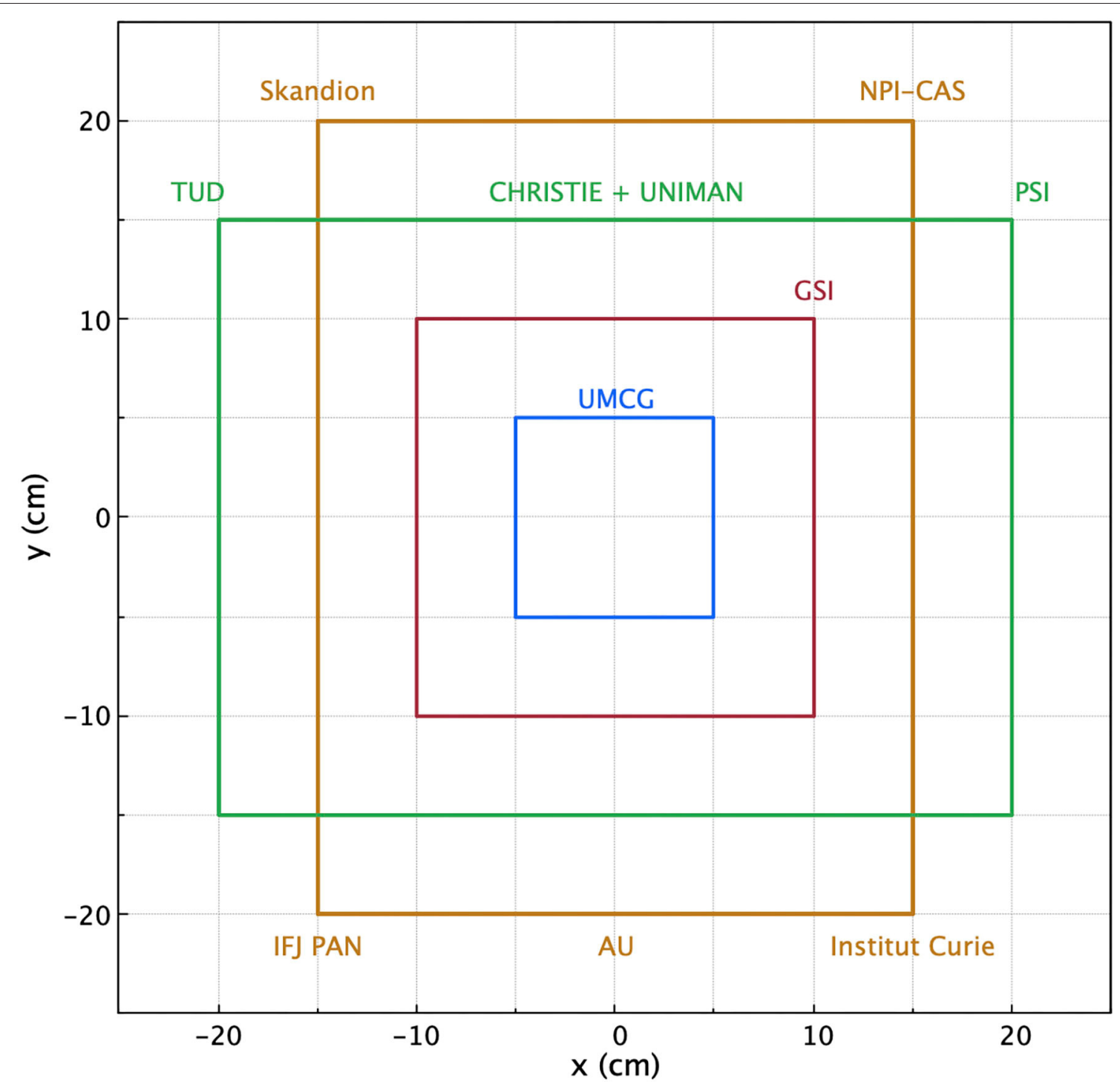

FIGURE 5 | Maximum available scanned field sizes of the INSPIRE partners, ranging from $10 \times 10 \mathrm{~cm}^{2}$ to $30 \times 40 \mathrm{~cm}^{2}$. Larger field sizes may be available by introducing scatterers.

beamlines, employ automated sample handling. Here, the sample can remain in a horizontal orientation and is lifted up only when presented to the beam for irradiation. Automated sample handling also has the added benefits of improving repeatability and minimizing access to the irradiation room, increasing sample throughput. Four centers employ automated sample handling. All the centers have the capability of a horizontal beamline, though four can additionally offer a vertical beam direction, and six offer more irradiating angles by using gantries. The sample type that can be irradiated is a limitation defined by the system. Most centers have flexibility here, with all capable of irradiating at least flasks and well-plates. The sample type capability may go beyond this (as long as it can be fixed in front of the beam and meet the safety regulations of the experimental room) and should be further discussed with the partner institute. Table 2 shows a summary of these details.
Figure 6 shows examples of sample presentation to the beam at Christie + UNIMAN, the research facility of UMCG, Institut Curie, GSI, and AU. The system at CHRISTIE + UNIMAN (Figure 6A) employs a 6-axis robot mounted inside a hypoxia end station. The space limitations of the hypoxia cabinet mean that at most a mix of up to 36 samples can be housed at a time. The fingers of the robot are designed for T75 flasks or 96-well-plates, limiting the sample type. However, other samples can be used so long as they have the same footprint as a 96-well-plate or through use of customized sample holders, alternatively a large range of samples can be used without the robot. Similar to the CHRISTIE + UNIMAN system, the GSI system (Figure 6D) holds samples in the horizontal position lifting them to the beam for irradiation. This change in orientation minimizes the time that cells are free from media, ensuring a good cellular environment and avoiding 
sample drying. Alternatively, samples can be prepared so that the culture vessel is full of cell media, which is the case for the research facility of UMCG (Figure 6B) and Institut Curie (Figure 6C).

\section{BIOLOGY - SAMPLE PREPARATION AND PROCESSING}

Alongside the physics capabilities, the biological equipment available at a center will often define the type and complexity of experiments that are possible. This impacts both the preirradiation sample preparation and post-irradiation analysis. For some experiments it is not possible to prepare samples prior to transport to the irradiating center. Similarly, it is not always possible to fix samples following irradiation ready for transport to the home institute. Table 3 gives details of the in vitro biological equipment available at INSPIRE partners. In most cases the equipment detailed in Table 3 is shared between the INSPIRE partner and other groups at the same institute. Therefore, these details should be used as a guide for maximum available equipment. Similarly, extra resources may be available at a partner's sister institute. Researchers requiring the use of any of this equipment should discuss their needs with the relevant partner.

Common amongst all centers is the availability of flow hoods and incubators, with TUD offering the largest capacity for sample preparation and storage. At the moment, only one center, UNIMAN, has a hypoxia station for irradiation of samples under variable oxygen tension. This offers the capability for studying the oxygen enhancement ratio and probing new fields such as the FLASH effect under strictly controlled conditions. The hypoxia station at UNIMAN is positioned directly at the beam nozzle, which prevents $\mathrm{O}_{2}$ fluctuations in the sample while it is being transported from the laboratory to the irradiation facility. Additionally, the irradiation in hypoxic conditions is possible at AU and GSI, where the samples can be gassed inside specially designed containers prior the transportation to the experimental room. The availability of more sophisticated post-irradiation analysis, such as flow cytometry, FACS, mass spectrometry, PCR, and sequencing is varied amongst the partners. Similarly, the advanced microscopy available amongst the partners is varied, though the majority have fluorescent and confocal microscopes available.

While all the INSPIRE TNA partners mentioned in this work offer the environment for in vitro studies, the in vivo capabilities are slightly more limited, as seen in Figure 2. Despite the data from cell experiments being a valuable preliminary tool for studying the effects of proton beams, all of the physiological processes and their complex interplay cannot be reproduced in vitro, and thus the clinical treatments must first be simulated using animal models before moving onto human trials. Table 4 shows the in vivo capabilities of the INSPIRE TNA providers.

In vivo experiments bring the added complexity of ethical review. INSPIRE has a well-established ethics platform for both its TNA and JRA, which is overseen by an ethics panel comprised of international experts in the field. The partners must also follow
TABLE 2 | Beamline and radiobiological sample details of the INSPIRE partners.

\begin{tabular}{|c|c|c|c|c|c|c|c|}
\hline \multirow[t]{2}{*}{ さ্ঠ } & \multirow[t]{2}{*}{ 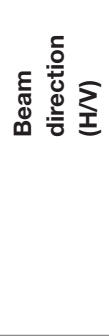 } & \multirow[t]{2}{*}{ 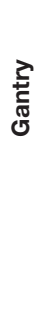 } & \multirow[t]{2}{*}{ 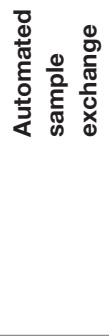 } & & \multicolumn{3}{|c|}{ Sample type } \\
\hline & & & & 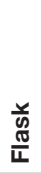 & $\begin{array}{l}\frac{5}{0} \\
\frac{10}{0} \\
\vdots \overline{0} \\
0 \\
0\end{array}$ & $\frac{\frac{0}{\pi}}{\frac{\pi}{0}}$ & 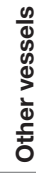 \\
\hline $\mathrm{AU}$ & $\mathrm{H}$ & $x$ & $x$ & $\checkmark$ & $x$ & $\checkmark$ & $\checkmark$ \\
\hline GSI & $\mathrm{H}$ & $x$ & $\checkmark$ & $\checkmark$ & $x$ & $\checkmark$ & $\checkmark$ \\
\hline IFJ PAN & $\mathrm{H}$ & $\checkmark$ & $x$ & $\checkmark$ & $\checkmark$ & $\checkmark$ & $\checkmark$ \\
\hline Institut Curie & $\mathrm{H}+\mathrm{V}$ & $\checkmark$ & $\checkmark$ & $\checkmark$ & $\checkmark$ & $\checkmark$ & $x$ \\
\hline NPI-CAS & $\mathrm{H}+\mathrm{V}$ & $\checkmark$ & $x$ & $\checkmark$ & $\checkmark$ & $\checkmark$ & $x$ \\
\hline PSI & $H+V$ & $\checkmark$ & $x$ & $\checkmark$ & $\checkmark$ & $\checkmark$ & $\checkmark$ \\
\hline UMCG & $\mathrm{H}$ & $x$ & $\checkmark$ & $\checkmark$ & $\checkmark$ & $\checkmark$ & $\checkmark$ \\
\hline Skandion & $H+V$ & $\checkmark$ & $x$ & $\checkmark$ & $\checkmark$ & $\checkmark$ & $\checkmark$ \\
\hline TUD & $\mathrm{H}$ & $x$ & $x$ & $\checkmark$ & $\checkmark$ & $\checkmark$ & $\checkmark$ \\
\hline CHRISTIE + & $\mathrm{H}$ & $\checkmark$ & $\checkmark$ & $\checkmark$ & $x$ & $\checkmark$ & $\checkmark$ \\
\hline
\end{tabular}
UNIMAN

All centers can offer a horizontal beamline, with the four able to irradiate samples from above or at user-defined angles using a gantry. There is flexibility in sample types, but the majority of centers have the ability to irradiate flasks and well-plates.

both the official regulations of their country/state as well as those of the TNA provider. Moreover, these regulations might vary from one state to another within the same country (for example, in Germany). Ethics applications in EU generally require a FELASA (Federation of European Laboratory Animal Science Associations) certification for participating scientists that cover the duration of the relevant research. In addition to that, countryspecific licenses might be required. In the latter case, exceptions can be made when the guest scientists are only irradiating the animals without leaving them at the TNA facility. The application for the ethical approval is normally done well in advance, as the review procedure can last up to several months. All of the paperwork relating to ethical approval is retained by the partner and made available to the EU upon request. In addition, for some experiments the EU requires copies of the ethical permissions prior to any experiment taking place.

\section{FUTURE PERSPECTIVES}

As has been shown, the resources available within the INSPIRE network are state-of-the-art. Further to this a number of new centers are under development and will soon be accessible to the research community. For example, the Proteus ONE IBA center at Charleroi (Belgium) will offer both in vitro and in vivo capabilities complete with a basic in vitro lab and animal facility on site, with researcher access offered through partnership with Namur. Belgium is also developing a center at Leuven, which will also offer in vitro and in vivo research capabilities. Furthermore, the European 

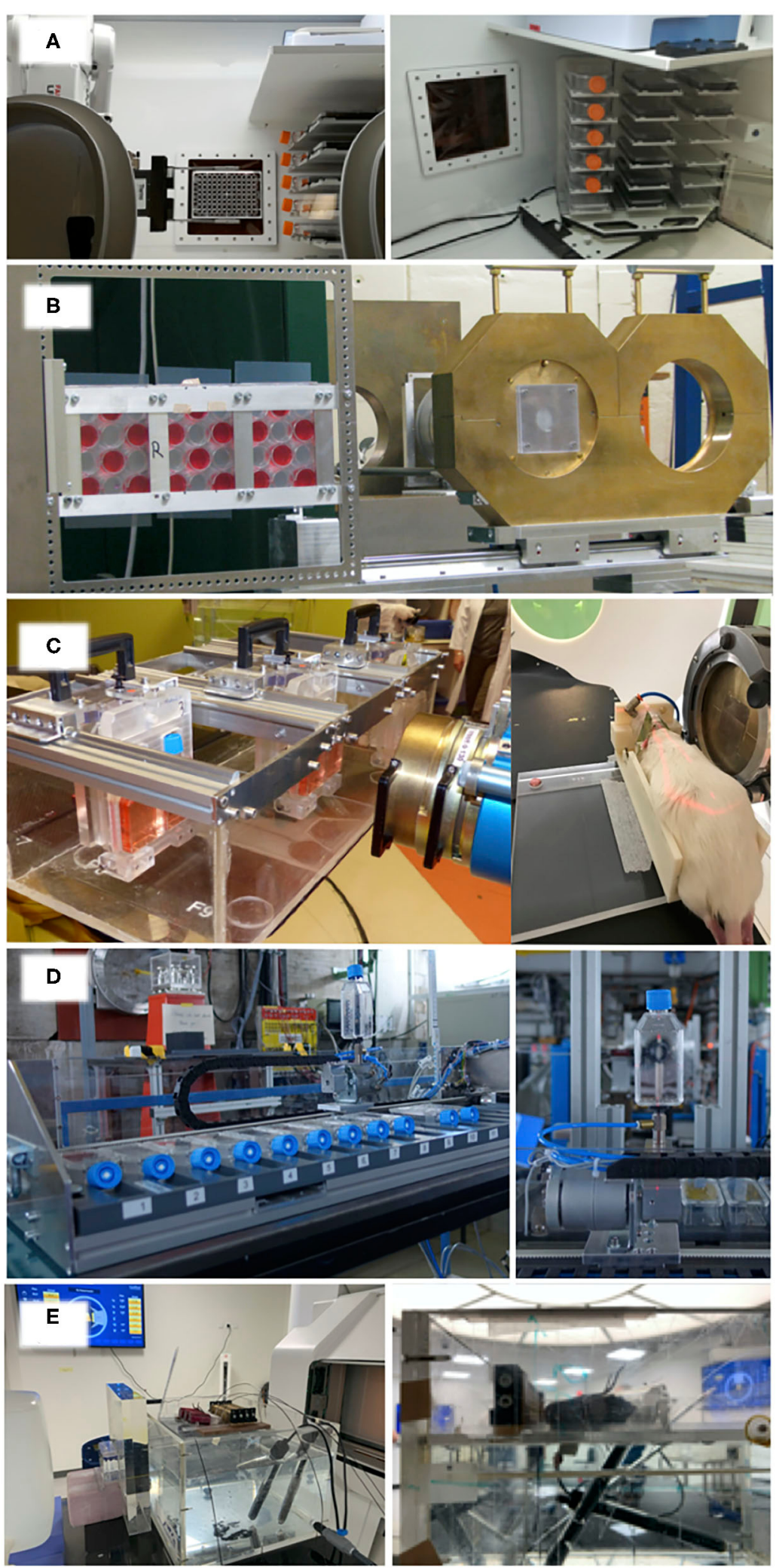

FIGURE 6 | Setup for sample irradiation at (A) CHRISTIE + UNIMAN, (B) UMCG research facility, (C) Institut Curie, (D) GSI, and (E) AU. The CHRISTIE + UNIMAN system is a 6 -axis robotic arm mounted in a hypoxia cabinet, allowing irradiation at different oxygen tensions from 0.1 to $20 \%$. The robot picks samples from a "hotel" and holds them in front of a beam window within the cabinet, before either replacing the sample to the hotel or moving to an automated fixation system (left). The hotel 
FIGURE 6 | can house up to 36 samples, a mix of T75 flasks or 96-well-plates (right). The system of the UMCG research facility shows the sequential irradiation of three 12-well-plates. Wells are filled with cell media and sealed with parafilm. The Institut Curie system shows sequential irradiation of six in vitro samples (left), and immobilized in vivo irradiation (right). The GSI system allows for sequential irradiation of 16 tissue culture flasks. The flasks remain in the horizontal position whilst not being irradiated (left), preventing the cell layer inside from drying. The robotic system lifts the sample and presents it to the beam (right), replacing it when irradiation is complete. The AU system shows an in vivo setup for mouse leg irradiation.

TABLE 3 | In vitro biological analysis equipment available at the INSPIRE partners.

\begin{tabular}{|c|c|c|c|c|c|c|c|c|c|c|c|c|}
\hline $\begin{array}{l}\frac{\bar{\Phi}}{\stackrel{D}{0}} \\
0\end{array}$ & 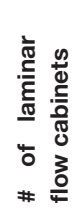 & 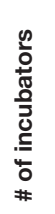 & 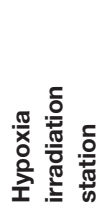 & 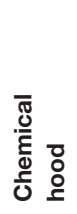 & 은 & ఝ্ড & 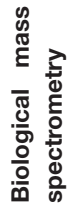 & 뜽 & 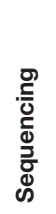 & 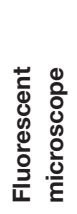 & 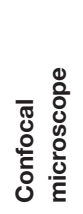 & 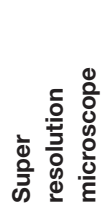 \\
\hline$A U$ & 1 & 1 & $x$ & $x$ & $x$ & $x$ & $x$ & $\checkmark$ & $x$ & $x$ & $\checkmark$ & $x$ \\
\hline Institut curie & 1 & 1 & $x$ & $\checkmark$ & $\checkmark$ & $\checkmark$ & $\checkmark$ & $\checkmark$ & $\checkmark$ & $\checkmark$ & $\checkmark$ & $\checkmark$ \\
\hline NPI-CAS & 2 & 3 & $x$ & $\checkmark$ & $x$ & $x$ & $x$ & $\checkmark$ & $\checkmark$ & $\checkmark$ & $x$ & $x$ \\
\hline $\mathrm{PSI}^{*}$ & 0 & 1 & $x$ & $x$ & $x$ & $x$ & $x$ & $x$ & $x$ & $x$ & $x$ & $x$ \\
\hline UMCG & 2 & 2 & $x$ & $\checkmark$ & $\checkmark$ & $\checkmark$ & $\checkmark$ & $\checkmark$ & $\checkmark$ & $\checkmark$ & $\checkmark$ & $x$ \\
\hline Skandion & 4 & 4 & $x$ & $\checkmark$ & $\checkmark$ & $\checkmark$ & $\checkmark$ & $\checkmark$ & $\checkmark$ & $\checkmark$ & $\checkmark$ & $\checkmark$ \\
\hline
\end{tabular}

"Biological equipment at PSI is available at a partner institute and will need to be discussed.

TABLE 4 | In vivo capabilities available at the INSPIRE partners.

\begin{tabular}{|c|c|c|c|c|c|c|c|c|}
\hline 㐫 & 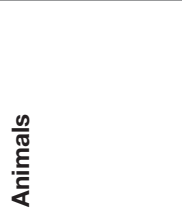 & 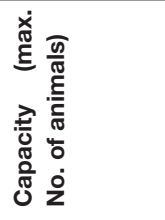 & 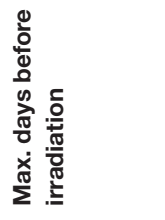 & 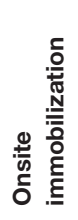 & 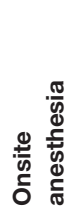 & 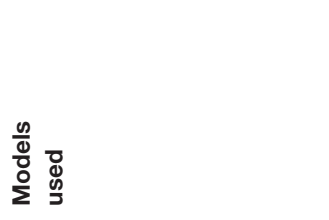 & 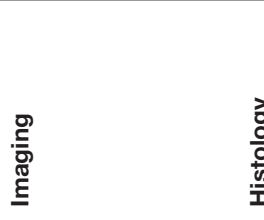 & 흥 \\
\hline $\mathrm{AU}$ & Rats, mice & $\begin{array}{l}80 \text { rats, } \\
200 \text { mice }\end{array}$ & 7 & $x$ & $x$ & $\begin{array}{l}\text { Normal tissue and a range of } \\
\text { tumor models (syngenic and } \\
\text { xenografts) }\end{array}$ & $x$ & $x$ \\
\hline GSI & Rats, mice & 80 & 7 & $x$ & $x$ & $x$ & $x$ & $\checkmark$ \\
\hline IFJ PAN & $\begin{array}{l}\text { Rats, mice, } \\
\text { hamsters }\end{array}$ & 100 & 7 & $\checkmark$ & $\checkmark$ & $x$ & MRI & $x$ \\
\hline Institut Curie & Rats, mice & $\begin{array}{l}100 \text { rats, } \\
40 \text { mice }\end{array}$ & A few months & $\checkmark$ & $\checkmark$ & $\begin{array}{l}\text { Normal tissue and a range of } \\
\text { tumor models (syngenic and } \\
\text { xenografts), orthotopic grafts, } \\
\text { specific tissue toxicity assays }\end{array}$ & $\begin{array}{l}\mathrm{CT}, \mathrm{X} \text {-ray, OCT, } \\
\text { Bioluminescence }\end{array}$ & $\checkmark$ \\
\hline TUD & $\begin{array}{l}\text { Rats, mice, } \\
\text { zebrafish }\end{array}$ & 100 & 7 & $\checkmark$ & $\checkmark$ & $\begin{array}{l}\text { Zebrafish embryo strain wild } \\
\text { type AB; NMRI nu/nu Nude, } \\
\text { C57BI/6JRj and C3H/HeNRj }\end{array}$ & $\begin{array}{l}\mathrm{CT}, \mathrm{X} \text {-ray, MRI, Proton } \\
\text { radiography, } \\
\text { Bioluminescence, PET, } \\
\text { Ultrasound }\end{array}$ & \\
\hline
\end{tabular}

"In vivo irradiation at PSI has previously been done, but capacities and equipment need to be discussed.

project SEEIST [38, 43] will develop capabilities in Southeastern Europe, filling in some geographical gaps shown in Figure 1. As well as developing a new heavy ion center the SEEIST project will have access to resources provided by INSPIRE.
There is a growing European interest into studying the effectiveness of heavy ions, with four operational carbon centers and two new centers under construction. A 2019 meeting of UK clinicians, scientists, engineers, and stakeholders began the process of considering future UK development of heavy ion 
therapy. There are also ongoing investigations into the clinical utilization of other particle types. For example, Helium has been seen as an intermediate between protons and carbon [44-46]. Other studies investigate the possibilities of combining multiple beams within one treatment plan to ensure a more uniform RBE distribution [47], or better treatment of hypoxic tumors [48]. The INSPIRE network is well-placed for the associated radiobiological investigations here, in particular with the partner institutes GSI and UMCG.

There has been a worldwide renewed interest in radiotherapy delivery techniques and improved normal tissue sparing. For example, spatially fractionated proton therapy [49-52] and ultra-high dose rate (FLASH) [53-56]. In these cases, the radiobiological mechanism driving the effect remains elusive. In particular, the differences between photon and particle therapy requires further investigation. Alongside this, the combination of particle therapy with immunotherapy $[57,58]$ is an exciting treatment that requires mechanistic understanding. Again, the INSPIRE network provides resources for investigation here, particularly through in vivo work, with results being directly useful for clinical adoption.

In vivo radiobiological research is a crucial step along the path to clinical implementation. Seven of the 11 partners discussed in this work are currently performing in vivo research (AU, GSI, IFJ PAN, Institut Curie, PSI, TUD, UMCG). Further to this, CHRISTIE + UNIMAN are beginning development of a second beamline for in vivo work. Skandion are also in the early stages of planning future in vivo work. This added capacity, and the currently available capacity, is sure to aid in the clinical efficacy of proton therapy.

The connection between research activities and clinically relevant questions must be made stronger. There are close links between many INSPIRE partners and clinical centers, which aids in this connection. However, it is important that the clinical community become more involved with research at inception. With a limited amount of finances this will ensure prioritization of the most pertinent research and advance clinical translation, all for the benefit of the patient.

\section{CONCLUSION}

In this work we have given details about the radiobiological capabilities of partners involved in the INSPIRE project, including how the resources can be accessed. It is clear that whilst there are a number of differences between the partners there are also a number of similarities. This allows for investigations into the cause of variance in published radiobiological data, such as the planned joint experiment of the INSPIRE partners. However, establishing these research centers requires significant investment and, as can be seen, many of the capabilities are already in place. More effort must be made to develop and utilize the resources currently available to us. Efforts are being made to further increase in vivo capabilities, whilst in vitro research is invaluable for identifying and probing mechanisms, in vivo research is crucial for clinical adoption. Also required here is a closer relationship with clinical partners, ensuring a good direction for future research. With a renewed interest in radiotherapy delivery techniques, and the unknown biological mechanisms, now is certainly and exciting time for particle radiobiology. Mechanisms that the INSPIRE network is well-placed to address.

\section{AUTHOR CONTRIBUTIONS}

$\mathrm{MDu}$ designed the structure of the manuscript. $\mathrm{NH}$ and $\mathrm{OS}$ wrote the manuscript with input from the other authors. OS and $\mathrm{MDu}$ provided information for GSI. LD and FP provided information for Institut Curie. JM and PO provided information for IFJ PAN. SB, MG, LB, MT, and JL provided information for UMCG. MDa and VV provided information for NPI-CAS. EB and JP provided information for TUD. $\mathrm{AL}$ and DCW provided information for PSI. AD and BS provided information for Skandion. PP, BSS, CG, and MS provided information for AU. A-CH and SL provided information for Namur. NH, JW, MM, RM, and KK provided information for UNIMAN and CHRISTIE. MDu leads the radiobiology work package of the INSPIRE project. KK leads the INSPIRE project. All authors reviewed and agreed the manuscript.

\section{FUNDING}

This work was funded by the European Union's Horizon 2020 research and innovation programme under grant agreement no 730983 (INSPIRE).

\section{REFERENCES}

1. PTCOG. Particle Therapy Facilities in Operation. (2020). Available online at: https://www.ptcog.ch/ (accessed May 11, 2020)

2. Grau C, Durante M, Georg D, Langendijk JA, Weber DC. Particle therapy in Europe. Mol Oncol. (2020) 14:1492-9. doi: 10.1002/1878-0261.12677

3. Durante M, Orecchia R, Loeffler JS. Charged-particle therapy in cancer: clinical uses and future perspectives. Nat Rev Clin Oncol. (2017) 14:48395. doi: 10.1038/nrclinonc.2017.30

4. Bortfeld TR, Loeffler JS. Three ways to make proton therapy affordable. Nature. (2017) 549:451-3. doi: 10.1038/549451a

5. Durante M. Proton beam therapy in Europe: more centres need more research. Br J Cancer. (2018) 120:777-8. doi: 10.1038/s41416-018-0329-x

6. Patriarca A, Fouillade C, Auger M, Martin F, Pouzoulet F, Nauraye C, et al. experimental set-up for flash proton irradiation of small animals using a clinical system. Int J Radiat Oncol. (2018) 102:61926. doi: 10.1016/j.ijrobp.2018.06.403

7. Calugaru V, Nauraye C, Noël G, Giocanti N, Favaudon V, MégninChanet F. Radiobiological characterization of two therapeutic proton beams with different initial energy spectra used at the institut curie proton therapy center in orsay. Int $J$ Radiat Oncol Biol Phys. (2011) 81:1136-43. doi: 10.1016/j.ijrobp.2010. 09.003

8. Calugaru V, Nauraye C, Cordelières FP, Biard D, De Marzi L, Hall J, et al. Involvement of the artemis protein in the relative biological efficiency observed with the 76-mev proton beam used at the institut curie proton 
therapy center in orsay. Int J Radiat Oncol Biol Phys. (2014) 90:3643. doi: 10.1016/j.ijrobp.2014.05.018

9. Beyreuther E, Baumann M, Enghardt W, Helmbrecht S, Karsch L, Krause $M$, et al. Research facility for radiobiological studies at the university proton therapy dresden. Int J Part Ther. (2018) 5:17282. doi: 10.14338/IJPT-18-00008.1

10. Müller J, Schürer M, Neubert C, Tillner F, Beyreuther E, Suckert T. Multimodality bedding platform for combined imaging and irradiation of mice. Biomed Phys Eng Express. (2020) 6:037003.

11. Suckert T, Müller J, Beyreuther E, Azadegan B, Brüggemann A, Bütof R, et al. High-precision image-guided proton irradiation of mouse brain sub-volumes. Radiother Oncol. (2020) 146:205-12. doi: 10.1016/j.radonc.2020.02.023

12. Szabó ER, Brand M, Hans S, Hideghéty K, Karsch L, Lessmann E, et al. Radiobiological effects and proton RBE determined by wildtype zebrafish embryos. PLoS ONE. (2018) 13:e0206879. doi: 10.1371/journal.pone.0206879

13. Beyreuther E, Brand M, Hans S, Hideghéty K, Karsch L, Leßmann E, et al, Feasibility of proton FLASH effect tested by zebrafish embryo irradiation. Radiother Oncol. (2019) 139:46-50. doi: 10.1016/j.radonc.2019.06.024

14. Müller J, Neubert C, von Neubeck C, Baumann M, Krause M, Enghardt W, et al. Proton radiography for inline treatment planning and positioning verification of small animals. Acta Oncol. (2017) 56:1399-405. doi: 10.1080/0284186X.2017.1352102

15. Kormoll T, Duplicy A, Enghardt W, Helmbrecht S, Hueso Gonzalez F. 106: A beam control system for an experimental beam line operated parallel to a therapeutic beam line. Radiother Oncol. (2014) 110:S523. doi: 10.1016/S0167-8140(15)34127-X

16. Helmbrecht S, Baumann M, Enghardt W, Fiedler F, Krause M, Lühr A. Design and implementation of a robust and cost-effective doublescattering system at a horizontal proton beamline. J Instrum. (2016) 11:T11001. doi: 10.1088/1748-0221/11/11/T11001

17. Bogdali-Suślik AM, Rawojć K, Miszczyk J, Panek A, Wozniak M, Szewczyk $\mathrm{K}$, et al. Influence of therapeutic proton beam on glioblastoma multiforme proliferation index - A preliminary study. Acta Phys Pol A. (2020) 137:649. doi: 10.12693/APhysPolA.137.64

18. Brandenburg S, Ostendorf R, Hofstee M, Kiewiet H, Beijers H. The irradiation facility at the AGOR cyclotron. Nucl Instruments Methods Phys Res Sect B Beam Interact Mater Atoms. (2007) 261:82-5. doi: 10.1016/j.nimb.2007.04.304

19. van Goethem M-J, Niemantsverdriet M, Brandenburg S, Langendijk JA, Coppes RP, van Luijk P. Development of a facility for highprecision irradiation of cells with carbon ions. Med Phys. (2011) 38:25663. doi: $10.1118 / 1.3528164$

20. Nagle PW, Hosper NA, Ploeg EM, van Goethem M-J, Brandenburg S, Langendijk JA, et al. The in vitro response of tissue stem cells to irradiation with different linear energy transfers. Int J Radiat Oncol Biol Phys. (2016) 95:103-11. doi: 10.1016/j.ijrobp.2016.02.020

21. Bijl HP, van Luijk P, Coppes RP, Schippers JM, Konings AWT, van der Kogel AJ. Influence of adjacent low-dose fields on tolerance to high doses of protons in rat cervical spinal cord. Int J Radiat Oncol Biol Phys. (2006) 64:1204-10. doi: 10.1016/j.ijrobp.2005.06.046

22. van Luijk P, Novakova-Jiresova A, Faber H, Schippers JM, Kampinga $\mathrm{HH}$, Meertens $\mathrm{H}$, et al. Radiation damage to the heart enhances early radiation-induced lung function loss. Cancer Res. (2005) 65:650911. doi: 10.1158/0008-5472.CAN-05-0786

23. Nagle PW, van Goethem M-J, Kempers M, Kiewit H, Knopf A, Langendijk JA, et al. In vitro biological response of cancer and normal tissue cells to proton irradiation not affected by an added magnetic field. Radiother Oncol. (2019) 137:125-9. doi: 10.1016/j.radonc.2019.04.028

24. van Luijk P, Pringle S, Deasy JO, Moiseenko V V, Faber H, Hovan A, et al. Sparing the region of the salivary gland containing stem cells preserves saliva production after radiotherapy for head and neck cancer. Sci Transl Med. (2015) 7:305ra147. doi: 10.1126/scitranslmed.aac4441

25. Merk B, Voss KO, Müller I, Fischer BE, Jakob B, Taucher-Scholz G, et al. Photobleaching setup for the biological end-station of the darmstadt heavyion microprobe. Nucl Instruments Methods Phys Res Sect B Beam Interact Mater Atoms. (2013) 306:81-4. doi: 10.1016/j.nimb.2012.11.043

26. Abdollahi E, Taucher-Scholz G, Durante M, Jakob B. Upgrading the GSI beamline microscope with a confocal fluorescence lifetime scanner to monitor charged particle induced chromatin decondensation in living cells. Nucl
Instruments Methods Phys Res Sect B Beam Interact Mater Atoms. (2015) 365:626-30. doi: 10.1016/j.nimb.2015.07.005

27. Sokol O, Scifoni E, Tinganelli W, Kraft-Weyrather W, Wiedemann J, Maier A, et al. Oxygen beams for therapy: advanced biological treatment planning and experimental verification. Phys Med Biol. (2017) 62:7798813. doi: $10.1088 / 1361-6560 / a a 88 a 0$

28. Graeff C, Lüchtenborg R, Eley JG, Durante M, Bert C. A 4D-optimization concept for scanned ion beam therapy. Radiother Oncol. (2013) 109:41924. doi: $10.1016 /$ j.radonc.2013.09.018

29. Schuy C, Tessa C La, Horst F, Rovituso M, Durante M, Giraudo $\mathrm{M}$, et al. Experimental assessment of lithium hydride's space radiation shielding performance and monte carlo benchmarking. Radiat Res. (2018) 191:154. doi: 10.1667/RR15123.1

30. Pleskac R, Abou-Haidar Z, Agodi C, Alvarez MAG, Aumann T, Battistoni G, et al. The FIRST experiment at GSI. Nucl Instruments Methods Phys Res Sect A Accel Spectrometers Detect Assoc Equip. (2012) 678:13038. doi: 10.1016/j.nima.2012.02.020

31. Bert C, Gemmel A, Saito N, Chaudhri N, Schardt D, Durante M, et al. Dosimetric precision of an ion beam tracking system. Radiat Oncol. (2010) 5:61. doi: 10.1186/1748-717X-5-61

32. Paganetti H. Relative biological effectiveness (RBE) values for proton beam therapy. Variations as a function of biological endpoint, dose, and linear energy transfer. Phys Med Biol. (2014) 59:R419-72. doi: 10.1088/0031-9155/59/22/R419

33. Friedrich T, Scholz U, Elsässer T, Durante M, Scholz M. Systematic analysis of RBE and related quantities using a database of cell survival experiments with ion beam irradiation. J Radiat Res. (2013) 54:494-514. doi: 10.1093/jrr/r rs114

34. Tommasino F, Durante M. Proton radiobiology. Cancers. (2015) 7:35381. doi: 10.3390/cancers7010353

35. Carabe A, Moteabbed M, Depauw N, Schuemann J, Paganetti H. Range uncertainty in proton therapy due to variable biological effectiveness. Phys Med Biol. (2012) 57:1159-72. doi: 10.1088/0031-9155/57/5/1159

36. Durante M, Paganetti H, Pompos A, Kry SF, Wu X, Grosshans DR. Report of a national cancer institute special panel: characterization of the physical parameters of particle beams for biological research. Med Phys. (2018) 46:e3752. doi: $10.1002 / \mathrm{mp} .13324$

37. Dosanjh M, Jones B, Pawelke J, Pruschy M, Sørensen BS. Overview of research and therapy facilities for radiobiological experimental work in particle therapy. report from the European particle therapy network radiobiology group. Radiother Oncol. (2018) 128:14-8. doi: 10.1016/j.radonc.2018. 03.008

38. Damjanovic S. SEEIIST: South East European International Institute for Sustainable Technologies. Europhys News. (2019) 50:31-2. doi: 10.1051/epn/2019404

39. Amaldi U, et al. South East European International Institute for Sustainable Technologies (SEEIIST). Front Phys. (2020).

40. Prall M, Durante M, Berger T, Przybyla B, Graeff C, Lang PM, et al. High-energy proton imaging for biomedical applications. Sci Rep. (2016) 6:27651. doi: $10.1038 /$ srep27651

41. RADIATE. Research And Development with Ion Beams - Advancing Technology in Europe.

42. Paganetti H, Blakely E, Carabe-Fernandez A, Carlson DJ, Das IJ, Dong $\mathrm{L}$, et al. Report of the AAPM TG-256 on the relative biological effectiveness of proton beams in radiation therapy. Med Phys. (2019) 46:e5378. doi: $10.1002 / \mathrm{mp} .13390$

43. SEEIST. The South East European International Institute for Sustainable Technologies.

44. Tessonnier T, Mairani A, Brons S, Sala P, Cerutti F, Ferrari A, et al. Helium ions at the heidelberg ion beam therapy center: comparisons between fluka monte carlo code predictions and dosimetric measurements. Phys Med Biol. (2017) 62:6784-803. doi: 10.1088/1361-6560/aa7b12

45. Knäusl B, Fuchs H, Dieckmann K, Georg D. Can particle beam therapy be improved using helium ions? - A planning study focusing on pediatric patients. Acta Oncol. (2016) 55:751-9. doi: 10.3109/0284186X.2015.1125016

46. Krämer M, Scifoni E, Schuy C, Rovituso M, Tinganelli W, Maier A, et al. Helium ions for radiotherapy? Physical and biological verifications of a novel treatment modality. Med Phys. (2016) 43:1995-2004. doi: 10.1118/1.4944593 
47. Kopp B, Mein S, Dokic I, Harrabi S, Böhlen TT, Haberer T, et al. Development and validation of single field multi-ion particle therapy treatments. Int J Radiat Oncol Biol Phys. (2020) 106:194-205. doi: 10.1016/j.ijrobp.2019. 10.008

48. Sokol O, Krämer M, Hild S, Durante M, Scifoni E. Kill painting of hypoxic tumors with multiple ion beams. Phys Med Biol. (2019) 64:045008. doi: 10.1088/1361-6560/aafe40

49. Prezado Y, Jouvion G, Patriarca A, Nauraye C, Guardiola C, Juchaux M, et al. Proton minibeam radiation therapy widens the therapeutic index for high-grade gliomas. Sci Rep. (2018) 8:16479. doi: 10.1038/s41598-018-34796-8

50. Henry T, Ödén J. Interlaced proton grid therapy - Linear energy transfer and relative biological effectiveness distributions. Phys Medica. (2018) 56:819. doi: 10.1016/j.ejmp.2018.10.025

51. González W, Prezado Y. Spatial fractionation of the dose in heavy ions therapy: An optimization study. Med Phys. (2018) 45:2620-27. doi: 10.1002/mp.12902

52. Prezado Y, Jouvion G, Hardy D, Patriarca A, Nauraye C, Bergs J, et al. Proton minibeam radiation therapy spares normal rat brain: long-term clinical, radiological and histopathological analysis. Sci Rep. (2017) 7:17. doi: 10.1038/s41598-017-14786-y

53. Vozenin M-C, De Fornel P, Petersson K, Favaudon V, Jaccard M, Germond J-F, et al. The advantage of flash radiotherapy confirmed in mini-pig and cat-cancer patients. Clin Cancer Res. (2018) 25:3542. doi: 10.1158/1078-0432.CCR-17-3375

54. Durante M, Brauer-Krisch E, Hill M. Faster and safer? FLASH ultra-high dose rate in radiotherapy. $\mathrm{Br} J$ Radiol. (2017) 91:20170628. doi: 10.1259/bjr.20170628
55. Favaudon V, Caplier L, Monceau V, Pouzoulet F, Sayarath M, Fouillade $\mathrm{C}$, et al. Ultrahigh dose-rate FLASH irradiation increases the differential response between normal and tumor tissue in mice. Sci Transl Med. (2014) 6:245ra93. doi: 10.1126/scitranslmed.3008973

56. Harrington KJ. Ultrahigh dose-rate radiotherapy: next steps for FLASH-RT. Clin Cancer Res. (2019) 25:3-5. doi: 10.1158/1078-0432.CCR-18-1796

57. Durante M, Brenner DJ, Formenti SC. Does heavy ion therapy work through the immune system? Int J Radiat Oncol Biol Phys. (2016) 96:9346. doi: 10.1016/j.ijrobp.2016.08.037

58. Durante M, Formenti S. Harnessing radiation to improve immunotherapy: better with particles? Br J Radiol. (2020) 93:20190224. doi: 10.1259/bjr.20190224

Conflict of Interest: The authors declare that the research was conducted in the absence of any commercial or financial relationships that could be construed as a potential conflict of interest.

Copyright (C) 2020 Henthorn, Sokol, Durante, De Marzi, Pouzoulet, Miszczyk, Olko, Brandenburg, van Goethem, Barazzuol, Tambas, Langendijk, Davídková, Vondráĉek, Bodenstein, Pawelke, Lomax, Weber, Dasu, Stenerlöw, Poulsen, Sørensen, Grau, Sitarz, Heuskin, Lucas, Warmenhoven, Merchant, Mackay and Kirkby. This is an open-access article distributed under the terms of the Creative Commons Attribution License (CC BY). The use, distribution or reproduction in other forums is permitted, provided the original author(s) and the copyright owner(s) are credited and that the original publication in this journal is cited, in accordance with accepted academic practice. No use, distribution or reproduction is permitted which does not comply with these terms. 